\title{
A New Method of B-spline Surface Reconstruction Using Hierarchical Surface
}

\author{
Lin Zizhi ${ }^{1,} a^{*}$, Ding Yun ${ }^{2, b}$, Shu Sihui ${ }^{1, c}$
}

1 College of Mathematics and computer Science, Jiangxi science \& Technology Normal University, Nanchang China

2 Jiangxi Normal University Science and Technology College, Nanchang China a linzizhi161@163.com, b8580090@qq.com, c 10108010@qq.com

Key words: Surface reconstruction; Parameterization; Best square approximation; Hierarchical surface

Abstract: In the process of surface reconstruction, the least squares fitting surface often produces singular matrix and it will cause a failure surface fitting. In this paper, we propose a new surface reconstruction method based on hierarchical surface. We first construct a low-order surface to interpolate data points, which is controlling surface, and then we use high-order surface to approximate the controlling surface, which is approximating surface. The best square approximation and hierarchical surface are used in this approximation to controlling surface, and we refine the control points mesh to approximate the controlling surface locally to achieve the given precision step by step. Finally, some examples of this algorithm demonstrate its usefulness and quality.

\section{INTRODUCTION}

Surface reconstruction to data points with the rectangular topology is the key problem in CAD/CAM [1-6]. Curved surface reconstruction problems in reverse engineering are inverted to go into the reconstruction of mesh data points, but the existing algorithms are lack of robustness and effective calculation in practical application [2]. Multiresolution surface method [2, 4, 5] provides a new way for the reconstruction of curved surface. Fossey [4, 5] in 1988 and 1995 have proposed the multi-level fitting methods, but control vertices when every time double insert node after encryption. Least squares fitting method is easy to produce singular matrix, so this paper proposes a new method for surface reconstruction. Only a parameterization can achieve data point's curved surface reconstruction in a given precision. In the process of reconstruction, use curved surface to get approximation data points as well as the approximation of curved surface at the same time to avoid the singularity problem of least squares fitting surface. Use a hierarchical surface grid through a control hierarchy network, from rough grid adaptive step by step to achieve the approximation accuracy. $C^{2}$ continuous is reached between the curved surfaces between different levels to realize multiresolution representation and analysis of curved surface.

\section{SURFACE RECONSTRUCTION}

\subsection{Surface approximation to mesh data}

Give a mesh of data point: $\left\{Q_{i, j}, i=1, \cdots m, j=1 \cdots n\right.$. $\}$. Find a $d_{u} \cdot d_{v}$ order B-spline surface:

$$
S(u, v)=\sum_{i=1}^{n_{u}} \sum_{j=1}^{n_{v}} S_{i, j} N_{i, d_{u}}(u) N_{j, d_{v}}(v), u \in[0,1], v \in[0,1]
$$

Where $S=\left\{S_{i, j}\right\}$ are control points. $\left\{N_{i, d_{u}}(u)\right\}_{i=1}^{n_{u}},\left\{N_{j, d_{v}}(v)\right\}_{j=1}^{n_{v}}$ are $d_{u}, d_{v}$ B-spline basis defined on the knot vectors $U=\left\{u_{1}, \cdots, u_{n_{u}+d_{u}}, u_{n_{u}+d_{u}+1}\right\}$ and $V=\left\{v_{1}, \cdots, v_{n_{v}+d_{v}}, v_{n_{v}+d_{v}+1}\right\}$, which makes $S$ (u, v) close to or through these data points. In order to show the convenience, express the curved surface as a one-dimensionalised form:

$$
S(u, v)=\sum_{i=1}^{n_{u}} \sum_{j=1}^{n_{v}} S_{i, j} N_{i}(u) N_{j}(v)=\sum_{k=1}^{n} S_{k} N_{k}(u, v)
$$


In it: $n=n_{u} \times n_{v}, S_{k}=S_{i(k), j(k)}, N_{k}(u, v)=N_{i(k)}(u) N_{j(k)}(v), i(k)=\left\lfloor\frac{k}{n_{v}}\right\rfloor+1, j(k)=k-(i(k)-1) n_{v}$

For the purposes of the behavior control of surface endpoint, under normal circumstances, take duplication of the ends vectors for the surface order that is:

$u_{1}=\cdots=u_{d_{u}+1}, u_{n_{u}+1}=\cdots=u_{n_{u}+d_{u}+1} ; v_{1}=\cdots=v_{d_{v}+1}, v_{n_{v}+1}=\cdots=v_{n_{v}+d_{v}+1}$

At present, the most commonly used data point fitting method is the least squares, namely after given the parameter values of data points and node vector, use the least square method to solve the control vertices for surface reconstruction, but in the process of surface high precision fitting, it is easy to appear strange phenomenon. This paper presents a new surface step by step fitting method based on hierarchy to avoid some shapes appeared in the process of fitting the mutation, interpolate all the data points to local structure curved surface interpolation; And then construct new surface close to the control surface. By two-step reconstruction method, turn data points surface reconstruction problem into two relatively simple fitting problems, and make surface shape under control. By constructing level surface to control surface in order to make the approximate surface have good quality and reasonable control vertices.

\subsection{The construction of controlling surface}

Local interpolation method has advantages such as simple structure, easy to modify, no need to consider singularity in the system and the shape of the surface can be controlled through local tangent vector etc., especially it is good for man-machine interactive surface reconstruction. Considering the above factors, this paper chooses $C^{1}$ continuous cubic B spline surfaces as control surfaces, which is also near the target surface. First of all, parameterize two-way average accumulative chord length on the number:

The corresponding parameter value of $\left\{Q_{k, l}, k=1 \cdots n, l=1 \cdots m\right\}$ is: $\left\{\left(u_{k}, v_{l}\right), k=1 \cdots m, l=1 \cdots n\right\}$. Local interpolation surface makes $S\left(u_{k}, v_{l}\right)=\sum_{i=1}^{2 n-1} \sum_{j=1}^{2 m-1} N_{i, 3}\left(u_{k}\right) N_{j, 3}\left(v_{l}\right) S_{i, j}$

Control surface node vector is as follows:

$U=\left\{0,0,0,0, u_{1}, u_{1}, \cdots, u_{n-1}, u_{n-1}, 1,1,1,1\right\}, V=\left\{0,0,0,0, v_{1}, v_{1}, \cdots, v_{n-1}, v_{n-1}, 1,1,1,1\right\}$

It can be seen that curved surface is made up of three Bezier surface of $(n-1) \times(m-1)$, and each piece structure is as follows:

1. The surface interpolation in the four corners $Q_{k, l}, Q_{k+1, l}, Q_{k, l+1}, Q_{k+1, l+1}$.

2. Calculate the four angle point's $u$ partial derivatives vector, and respectively calculate 2control vertices of $u$ boundary curves.

3. Calculate the four angle point's $v$ partial derivatives vector, and respectively calculate 2control vertices of $v$ boundary curves.

4. Calculate the four corners mixed partial derivative vector, and calculate the four control points of internal surface.

The calculation method of partial derivative vector and mixed partial derivative vector are calculated according to three point method [6], and specific process structure can be seen in reference [6]. Control surface automatically to keep G1 continuous set up in the process. 


\subsection{B-spline surface approximation to the controlling surface}

After established control surface, due to control surface interpolates in data points; we just need to build another surface for approximation data points as well as control surface. This paper adopts the following objectives as the two curved surface approximation degree method:

$$
E_{\text {dist }}=\iint\|S(u, v)-P(u, v)\|^{2} d_{u} d_{v}
$$

The objective function uses the function L2 norm to measure surfaces approximation error, in which $S(u, v)$ is the control surface, $P(u, v)$ is the approximate surface, and use type (1) defined surface representation. When the node vector is determined, we get an optimization variable system about control vertices. Only need to make n partial derivative of $P=\left\{P_{k}\right\}$ as zero E, $l=1,2, \cdots n$ and we get a system of linear equations about control vertex $P=\left(P_{1}, \cdots, P_{n}\right): M P=R$.

In it:

$$
M_{k, l}=\iint_{\Omega} N_{k}(u, v) \bullet N_{l}(u, v) d_{u} d_{v} \quad R_{l}=\iint_{\Omega} N_{l}(u, v) Q(u, v) d_{u} d_{v} \quad, \quad N_{k}(u, v), N_{l}(u, v)
$$

As type (1) defined, based on b-spline base local supporting, when $|i-j| \geq k, N_{i, k}(u) N_{j, k}(u)=0$, so $\mathrm{M}$ is nonzero sparse matrix of symmetry of each line with $(2 \mathrm{k}+1) *(2 l+1)$. After a given node vector, use B - spline base differential agreed righteousness, and expand it into a power base in form of matrix calculation $M=\left(M_{i, j}\right)$. Adopt the method of literature [7], we can quickly calculate the product of any two spline base integral values, which is more advantageous to reduce the error of curved surface approximation. After $\mathrm{M}$ and $\mathrm{R}$ are obtained, use Gaussian elimination method to solve the linear equations, obtain control vertices, so as to get approximate surface. If use solution for large sparse system linear equations, we can solve the problem of large scale of data points. Matrix $M$ has been proved to be positive definite matrix, and the concrete proof is similar to literature [7], so the best square approximation won't appear singular matrix, which can make more free choice on node vector march surface reconstruction.

\section{HIERARCHICAL SURFACE}

In fitting process for the first time, use average method [6] for surface node vector approximation and, of course, the first choice of node vector cannot satisfy the requirement of curved surface approximation precision, so in the area of error overrun, we use a finer control grid to control surface, in order to further reduce the approximation error. For error area, and there is no need to do further processing.

\section{1 The smooth connection between the hierarchical surfaces}

The new child surface produced next layer surface must be stitched with last layer father surface smoothly. Use methods of joining together in literature [1], extend from four directions respectively along the region to constitute an interval constraint, the constraint zone remains control vertices overlap of three lines of three columns with the father surface, keep the father surface and sub-surface C2 continuous, and the rest can make enough freedom for better approximate the target surface, set the method as below:

\subsection{Error calculation}

$\left\|Q_{i, j}-P\left(u_{i}, v_{j}\right)\right\|$ is adopted for representation of data point error. When the control of surface is relatively sparse, data points can produce approximation error. The fitting errors of the data points 
are concentrated in a small area, so it is not necessary to carry out surface global refining, but local reconstruction of curved surface.

In the localization algorithm of error overrun subdomain, first find any error overrun subdomain (error overrun data points in a node interval), and then search in eight adjacent direction. If we find any error overrun sub area, merge them. This process will be recursive, and the end condition is in any adjacent direction no error overrun sub area or reached the edge of father area. Because we have to scale out an area for the adjacent two sub areas, if their distance is only one interval, merge them into one area.

\subsection{The best square approximation with constrains}

In order to keep smooth joining together with the father area, after insert node area boundary, it is consistent with the three rows or columns of nodes, so in the process of local fitting, adopt the best square approximation with binding, the curved surface equation is divided into control vertices and the disturbances: $S(u, v)=\sum_{k=1}^{n} N_{k}(u, v)\left(P_{k}+\delta_{k}\right), P_{k}, k=1 \cdots n$ is control vertices after father surface insert node, $\delta_{k}, k=1 \cdots n$ is disturbance quantity of control vertices, the control vertices' disturbance quantity of three lines of three columns of border is zero, the best square approximation goal is to calculate control vertices' disturbance quantity $\delta_{k}$ of control area, namely:

$$
E_{\text {dist }}=\iint\left\|\sum_{k}\left(R_{k}+\delta_{k}\right) N_{k}(u, v)-\sum_{l} C_{l} \bar{N}_{l}(u, v)\right\|^{2} d_{u} d_{v}
$$

Finally it can be reduced to the following equation:

$\bar{M} r=N Q-M P$

$R$ is control vertices after insert node, $r$ is the disturbance vector, $M_{k, l}=\iint_{\Omega} N_{k}(u, v) \bullet N_{l}(u, v) d_{u} d_{v}, k=1 \cdots n, l=1 \cdots n$

$\bar{M}$ is $M$ 's node vector $U=\left\{u_{1}, u_{2}, u_{3}, u_{4} \cdots, u_{n_{u}+1}, u_{n_{u}+2}, u_{n_{u}+3}, u_{n_{u}+4}\right\}$,

$V=\left\{v_{1}, v_{2}, v_{3}, v_{4} \cdots, v_{n_{u}+1}, v_{n_{u}+2}, v_{n_{u}+3}, v_{n_{u}+4}\right\}$ node vector $\tilde{U}=\left\{u_{4} \cdots, u_{n_{u}+1}\right\}, \tilde{V}=\left\{v_{4} \cdots, v_{n_{u}+1}\right\}$

There is no need for recalculation.

$N_{k, l}=\iint_{\Omega} N_{k}(u, v) \bullet \bar{N}_{l}(u, v) d_{u} d_{v}, k=1 \cdots m, l=1 \cdots n$

Use constrained optimal approximation, and keep $C^{2}$ continuous with the father surface in sub surface fitting process automatically.

\subsection{The choice of sub area control surface}

Looking for areas which do not meet the error $\Omega=\left[u_{k u 1}, u_{k u 2}\right] \times\left[v_{k v 1}, v_{k v 2}\right]$, according to the continuity constraint, expand the error area up and down or so four directions for a grid, in the regions $\left[u_{k u 1+1}, u_{k u 2+1}\right] \times\left[v_{k v 1+1}, v_{k v 2+1}\right]$, make new curved surface fitting, so look for control area in curved surface, insert node $\left\{u_{k u 1+1}, u_{k u 2+1}, v_{k v 1+1}, v_{k v 2+1}\right\}$ to controlling surface respectively, find out the new control surface $S_{\text {new }}$ (new approximate the target surface) defined $\left[u_{k u 1+1}, u_{k u 2+1}\right] \times\left[v_{k v 1+1}, v_{k v 2+1}\right]$ surface. Implement the best square approximation based on constraint, and produce new fitting surface. 


\subsection{Control mesh refinement}

First fitting adopts average method to calculate the vectors for surface node approximation, and this method preliminarily reflects the distribution of data points, which establishes the father surface after closing to control surface. For after local curved surface fitting of each layer, insert nodes in order to increase surface degrees freedom to reduce surface approximation error. In each area fitting, adopt the method of double encryption, namely insert the midpoint in the area of each node in document vector interval between the area, and the number of control points is twice the number in the region.

\section{THE EXPERIMENT AND RESULT ANALYSIS}

This model is for some auto scan data. Figure 1 shows the original data points, and control surface control grid is shown in Figure 2. Due to interpolation data points, control surface data point is redundant. Figure 3 shows e control mesh surface approximation for the first time. Because the grid is sparse, and the error of data points is larger, Figure 4 shows a partial encryption control grid control grid after the fitting to reduce approximation error of data points. Figure 5 is the final surface reconstruction.
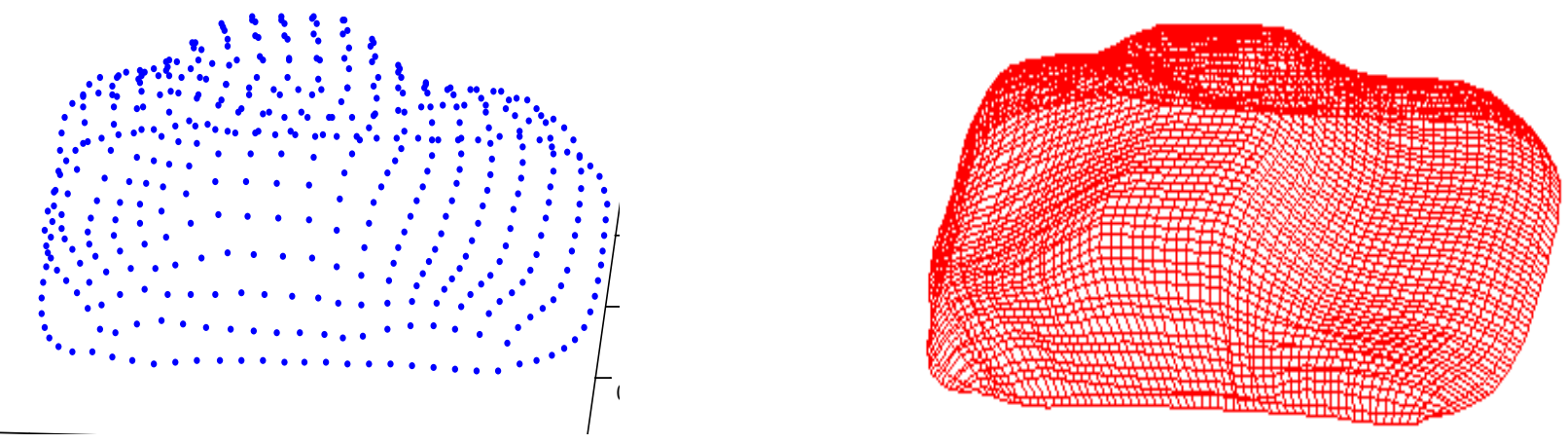

Fig1 The scanning mesh data points of a car Fig 2 The controlling surface interpolating data points

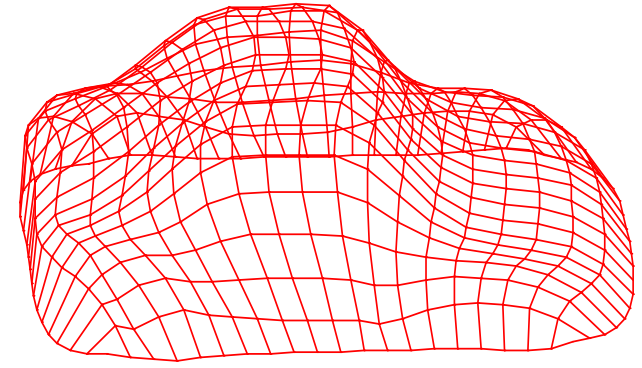

Fig 3. The approximating surface in first time

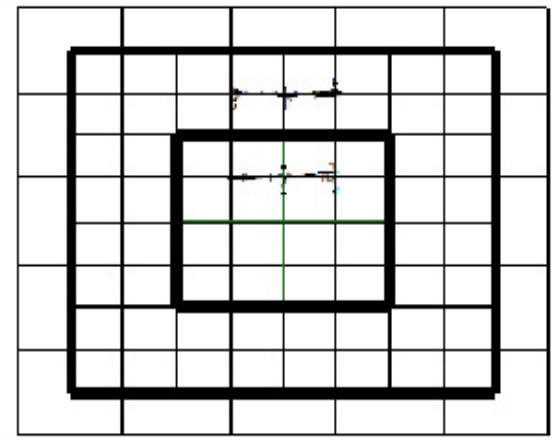

Fig 5. The refined knot mesh

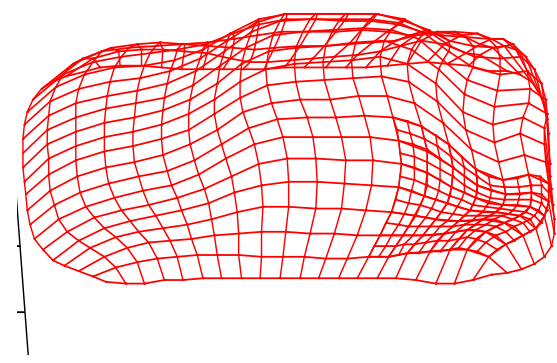

Fig 4 The local refined surface

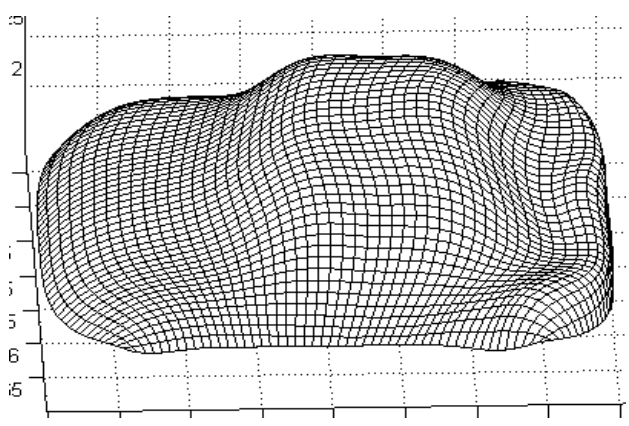

Fig 5. The final approximating surface 


\section{CONCLUSION:}

This article adopts interpolation approximation method for data reconstruction, and then through the hierarchy fitting technique, control grid approximation by gradual encryption, and implement the approximation of given data points error. This method has the following advantages:

1. Avoid singular phenomenon of surface reconstruction problem. By controlling the surface make near surface shape under control.

2. In the process of iterative fitting, there is no need for parameterized matters related to data points, but need to take out the corresponding part for control surface fitting.

3. The use of local editing techniques improves the computational efficiency, and realizes multiresolution representation of curved surface.

\section{ACKNOWLEDGMENT}

This work is financially supported by the Natural Science Foundation of the overall Province of China under Grant 20122 bab201004; The General Natural Science Foundation of the overall Science \& Technology University under Grant No. 2013 xjyb002 and the Twelfth Five - Year Guideline General Education Science Foundation of the overall Province No. 15 yb191.

\section{REFERENCES}

[1] Piegl L A, Tiller baron urface approximation to scanned data [J]. Journal of Visual Computer, 2000, 16:386-395

[2] Zhang Weiqiang, Tang Zesheng. B-spline surface expression of large-scale scattered data level [J]. Journal of Computer. 1999, 22 (10) : 1059-1064.

[3] Wu Jianjie, Wang Qifu, Huang Yunbao, etc. The research progress of surface reconstruction in reverse engineering [J]. Journal of Engineering Graphics, 2004, 25 (2) : 133-142

[4] Forsey D.R, Bartels r. h. Hierarchical B - spline refinement.Computer Graphics, 1988, 22 (4) : 205-212.

[5] Forsey D R, Bartels r. h. Surface fitting with hierarchical splines. The ACM Trans on Graphics, 1995, 14 (2) : 134-161.

[6] Piegl L A, Tiller W.T he NURBS book. [M].Milan: the Springer, 1997.

[7] Lin Zizhi, Pan Rijing. A kind of new method of B-spline curve approximation[C]. Proceedings of the 3rd geometric design and calculation. 2007.7. 